\title{
Online Face Detection and Recognition in a Video Stream
}

\author{
Maha A. Hasso Al-Ghurery \\ College of Computer Science and Mathematics \\ University of Mosul, Mosul, Iraq
}

Received on: 05/12/2012

Accepted on: 03/04/2012

\section{ABSTRACT}

Faces detection and recognition process received considerable attention during the past decade, it is still considered one of the most important studies in the field of Image Processing, Pattern Recognition, and Computer Vision, where it drew the attention of many researchers from both the academic and industrial environments because of the broad scope of its practical applications. Face recognition system is considered as one of the biometric information processing systems that is easy to apply, the necessity of identifying the identity in the areas of security and surveillance systems made face recognition system one of the most important biometric techniques used in the identification of the individual.

In this work a system is designed for Online Face Detection and Recognition depending on multiple algorithms that are: AdaBoost algorithm for the face detection and the two algorithms Principle Component Analysis (PCA) and Linear Discriminate Analysis (LDA) to extract features and use back propagation neural network in recognition.

This system has been applied on a group of people using different numbers of features extracted from the face. Good recognition ratios have been obtained reached $(87.5 \%)$ relying on the key frame in the calculation of the of recognition ratio and (94\%) relying on the outcome of the 25 video frames in the calculation of the of recognition ratio. Language.

The System was implemented using graphical interfaces of Visual Studio C\# 2010

Keywords: Face Detection, AdaBoost algorithm, Principle Component Analysis (PCA), Linear Discriminate Analysis (LDA), back propagation neural network.

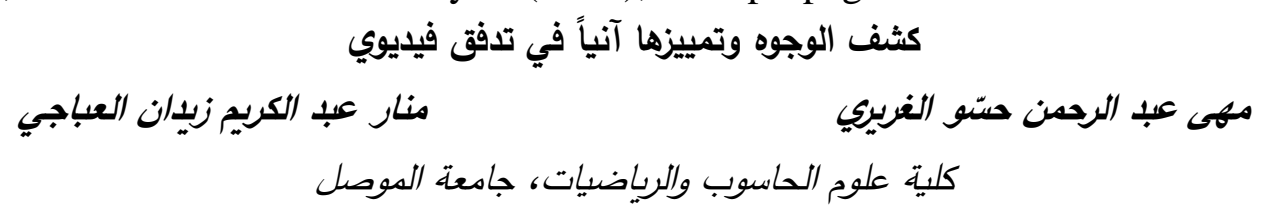

\footnotetext{
تاريخ قبول البحث: 2013104103
}

تاريخ استلام البحث: $2012 \backslash 12105$

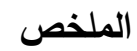

حَظِيت عملية كثف الوجوه و تمييزها اهتمامـا كبيرا خـلال العقد الماضي ولا يزال يُعد من أكثر الدراسات أهمية في مجال معالجة الصور والتعرف على الأنماط والرؤيا الحاسوبية إذ انها جذبت انتباه العديد من الباحثين من كلا البيئتين الأكاديمية والصناعية بسبب النطاق الواسع لها في كثير من التطبيقات العملية. ويُعد نظام تمييز

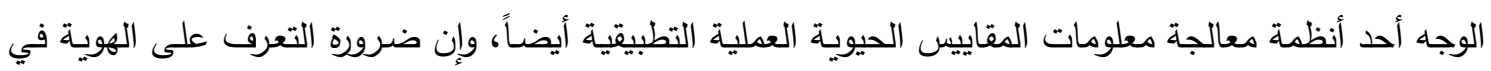

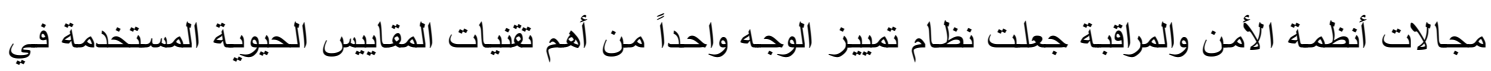
التعرف على هوية الفرد.

تم في هذا العمل تصميم نظام اعتماداً على خوارزميات متعددة لغرض كثف الوجوه وتمييزها بصورة آنية

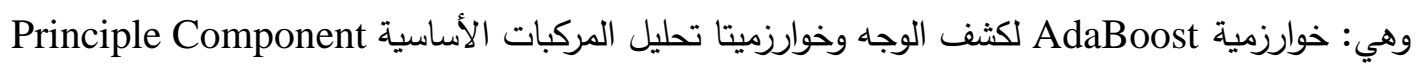


Analysis(PCA) واستخدام الثبكة العصبية ذات الانتشار الخلفي Back propagation Neural Network في التمييز.

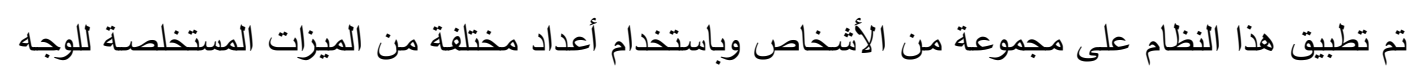

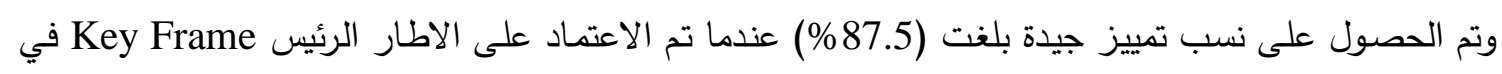

حساب نسبة التمييز و (94 \%) عندما تم الاعتماد على محصلة 25 اطار فيديو في حساب نسبة التمييز.

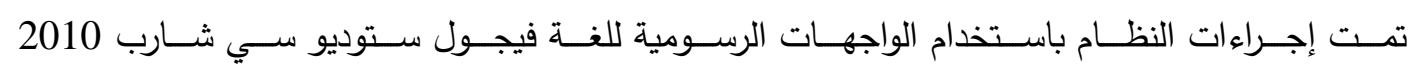

.(Visual Studio C\# 2010)

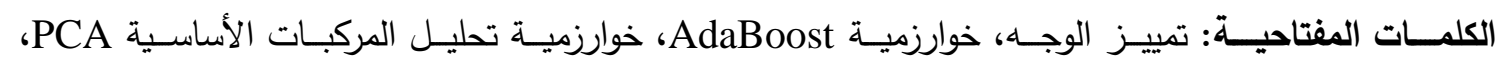

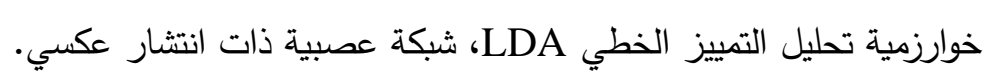
1 - 1 المقدمة

كثف الوجوه وتمييزها تملك العديد من التطبيقات من أهمها: المراقبة بالفيديو، والتحقيقات الجنائية وتطبيقاتها في الطب الثرعي، والخدمات المصرفية الإكترونية الآمنة، والهواتف النقالة، وبطاقات الائتمان، وتأمين

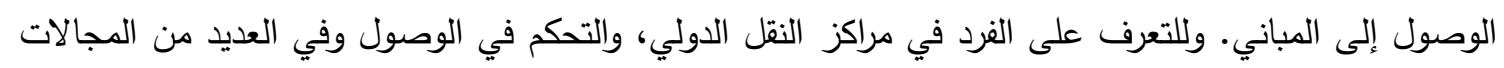

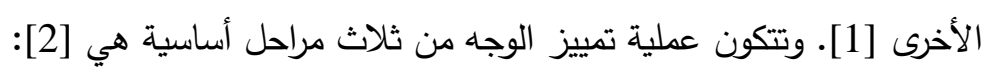

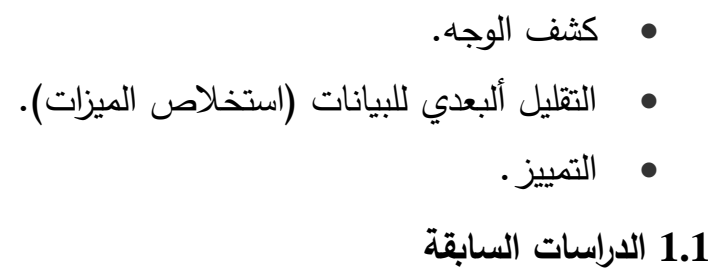

هناك عدة دراسات سابقة أجريت في مجال كثف الوجه وتمييزه في الصور الثابتة وكذلك في الصور المتحركة من خلال انظمة مراقبة فيديوية ومن اهمها: قام Jing Zhang وآخرون في عام (2008) [3 باقتراح

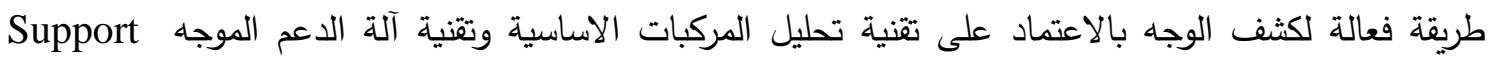
Vector Machine (SVM) التكراري, إذ أن كل من منطقة الوجه والمنطقة التي لا تحوي وجه تملك توزيع مدرج تكراري مختلف, ثم يتم استخدام طريقة PCA لتأكيد منطقة الوجه عن طريق تدريبها على 1000 أنموذج لصور وجوه ذات حجم 19 19 و اخيرا

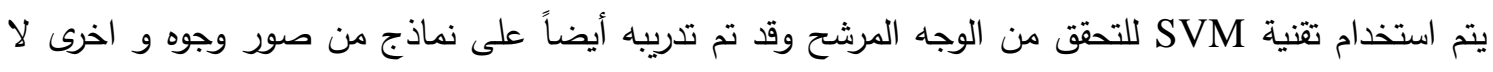

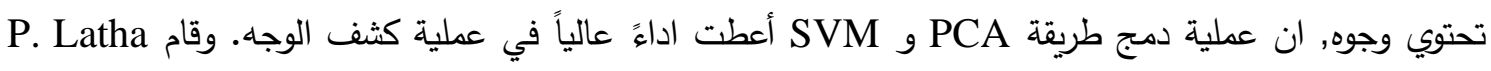

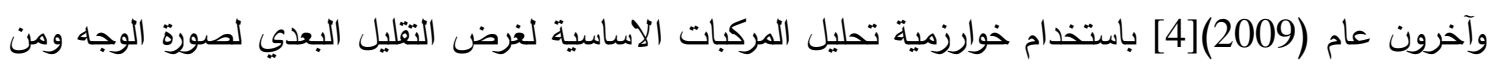
ثم استخدام الشبكة العصبية ذات التغذية الراجعة في عملية التمييز , وتم اختبار هذه الطريقة على صور وجوه من قاعدة بيانات Yale وبينت النتائج ان تمييز الوجه المعتمد على الشبكة العصبية صلب و ذو أداء جيد. كما قام Mayank Agarwal المركبات الأساسية في عملية استخلاص الميزات وتستخدم الثبكات العصبية في عملية التمييز , وبدلا من استخدام شبكة واحدة لجميع الأشخاص قام الباحث باستخدام شبكات عصبية عددها مساوٍ لعدد الأشخاص الذين يتم تمييز صورهم كل شبكة تدرب لتمييز شخص معين, والقرار النهائي للتمييز يعتمد على الإخراج لجميع الثبكات العصبية. 
وقام الباحث Wang-xiaoping عام (2011)[6] بتكوين نظام لكثف الوجه وتمييزه باستخدام المعلومات اللونية لتكوين أنموذج لون الجلد وهي أول مرحلة لتحديد موقع الوجه في الصورة وذلك بعد تحويل فضاء اللون من RGB الى YCrCb, ثم تأتي مرحلة فصل منطقة الجلد عن بقية المناطق بطريقة إحصائية وهي توزيع كاوسيان الثنائي البعد. اما في عملية التمييز فتم استخدام طريقة EigenFaces لاستخلاص الخواص الإحصائية والشبكة العصبية في عملية التصنيف. كما قام الباحثان Monadjemi S. A. Tofighi A عام (2011) [7 باقتئراح نظام صلب لكثف الوجه وتمييزه, في مرحلة كثف الوجه تم استخدام طريقة التقسيم بالاعتماد على لون الجلد مع خوارزمية AdaBoost لغرض كثف الوجه بدقة عالية, وتمت عملية استخلاص الخواص بواسطة مرشحات جابور Gabor Filters ومن ثم استخدام طريقة تحليل المركبات الاساسية للتقليل البعدي للبيانات, بعدها يتم اختيار الخواص باستخدام طريقة تحليل التمييز الخطي واخيرا استخدام تقنية SVM في عملية التصنيف.

2.1 كثف الوجه

كثف الوجه هو تقنية لتحديد الوجه البشري في صورة ما، وهو خطوة هامة في التعرف على الوجه ويُعد

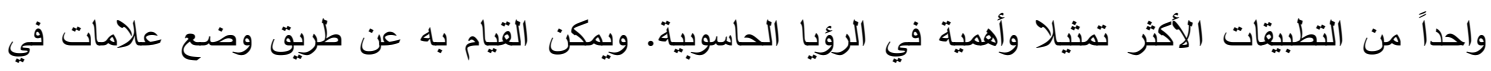

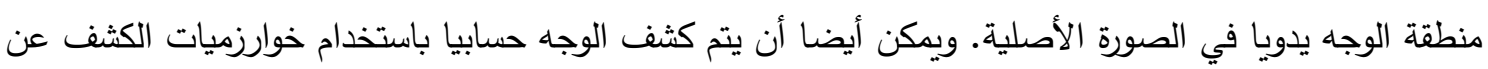
الوجه التي تتفذ عملية بحث عن الوجه وترجع مجموعة من المناطق ذات الاهتمام في الصورة الأصلية التي تحتوي

على الوجوه التي تم العثور عليها]2] • يتم تصنيف طرائق كثف الوجه في الصورة إلى أربعة أصناف هي [8]:

\section{Knowledge Based Methods}

1.2.1 طرائق معتمدة على المعرفة

يتم تطوير طرائق كثف الوجه في هذا الأسلوب بالاعتماد على قوانين مشتقة من معرفة الباحثين لوجوه

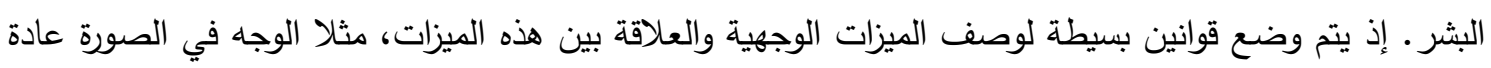

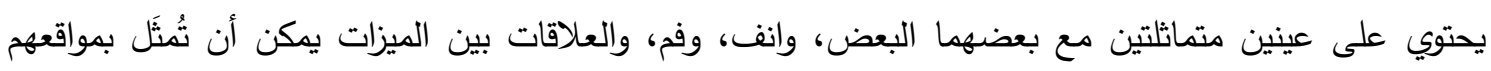
والبعد النسبي بينهم [9].

\section{Feature Invariant Methods}

2.2.1 طرائق الميزات الثابتة

تستخدم هذه الطرائق في إيجاد ميزات وجهية بنيوية لتحديد الوجوه و تعيين موقعها والتي تكون موجودة دائما

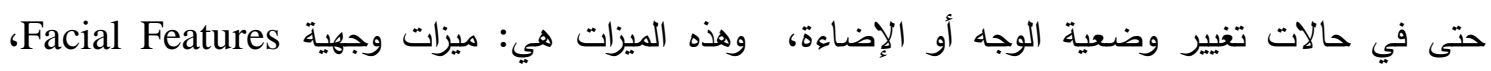
والنسيج Texture، ولون الجلد Skin Color، وميزات متعددة Multiple Features.

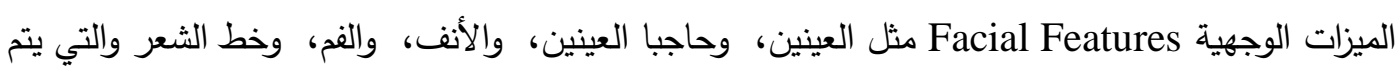
استخلاصها باستخدام طرائق اكتشاف الحواف Edge Detection وبالاعتماد على الميزات المستخلصة يتم بناء

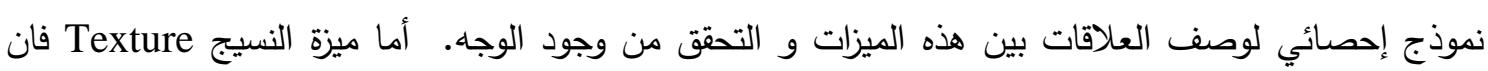

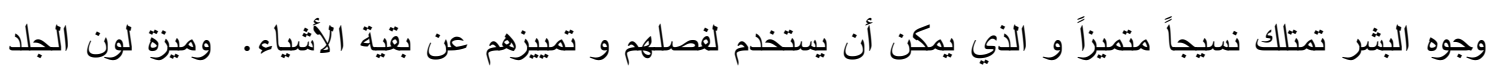
Skin Color

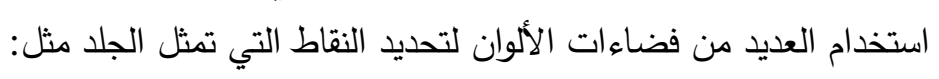

RGB(Red,Green,Blue), normalized RGB , HSV(Hue,Saturation, and Value) or HIS (Hue,Saturation, and Intensity), YIQ(Luminance-Chrominance), YCrCb(LuminanceChrominance), YES,CIE XYZ, CIE LUV. 
أما بالنسبة للميزات المتعددة Multiple Features فقد تم مؤخرا اقتراح طرائق تدمج العديد من الميزات واغلب هذه الطرائق تستخدم ميزات عامة مثل لون الجلد أو الثكل لإيجاد الوجوه المرشحة ثم استخدام ميزات محلية

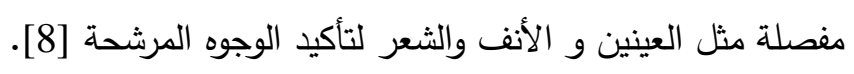

\section{Template Matching Methods} 3.2.1 طرائق ملانمة القالب

في ملائمة القالب يتم تكوين أنموذج وجه قياسي (عادة أنموذج وجه أمامي) يُكوّن يدوياً أو باستخدام دالة. فعند إدخال صورة يتم حساب قيم الارتباط Correlation Values مع الانموذج القياسي لكل من محيط الوجه، وادئ والعينين، والأنف، والفم بصورة مستقلة وبالاعتماد على قيم الارتباط يتم تحديد وجود أو عدم وجود وجه في الصورة.

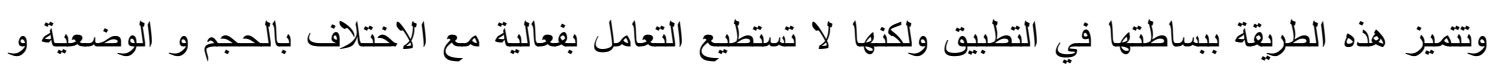

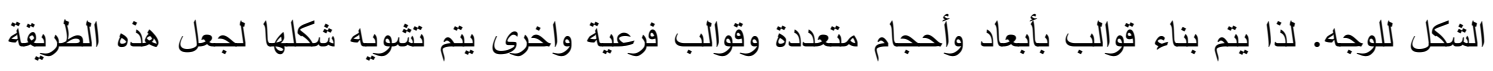
غير متأثرة بالحجم و الثكل [8].

\section{Appearance Based Methods}

4.2.1 الطرائق المعتمدة على الظهور

وهنا يتم تكوين مجموعة من القوالب (النماذج) عن طريق مجموعة من الصور المدربة وهذه النماذج المتكونة

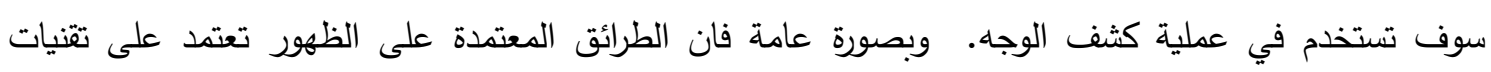

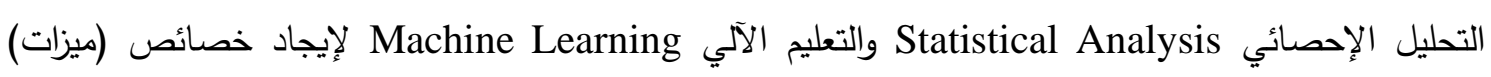
Principal Component صور الوجه و صور غير الوجه، من هذه الطرائق تحليل المركبات الأساسية الإسية Analysis ونماذج ماركوف المخفية Hidden Markov Model، وخوارزمية التدريب AdaBoost [10].

\section{Dimension Reduction Techniques} 3.1

أدت عمليات تحسين قدرة الحصول على البيانات والكلفة المنخفضة لآلية تخزين البيانات والتطور في تقنيات

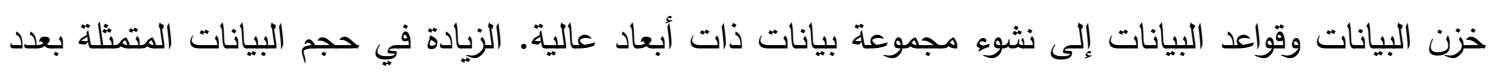

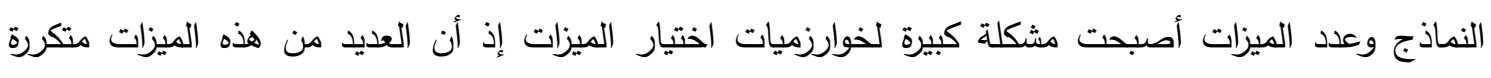

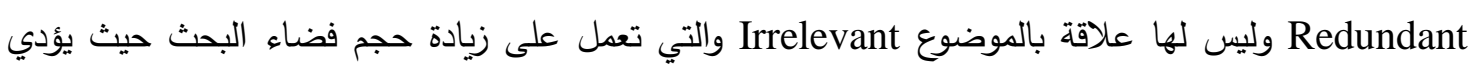
بدوره إلى صعوبة إجراء معالجة إضافية على البيانات. تمثل هذه الزيادة في الأبعاد عقبة هامة في تطبيقات التعلم الآلي والتتقيب عن البيانات، فضلاً عن أنها تتطلب مساحة خزنية ووقت معالجة كبيرين لذا نحتاج إلى إلى تقليل الأبعاد عن طريق تقنيات التقليل البعدي. ان الغرض الرئيسي من تقليل الأبعاد هو لتحسين أداء التصنيف عن طريق إزالة الميزات المكررة والتي ليست لها علاقة بالموضوع، ويمكن أن ينجز بطريقتين هما اختيار الميزات و استخلاص الميزات. اختيار الميزات

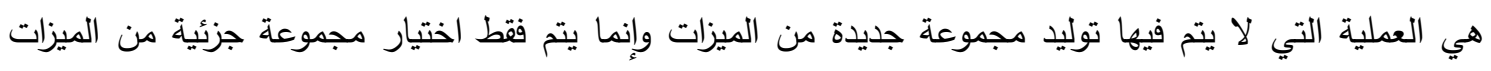
الأصلية مما يؤدي إلى التقليل البعدي. أما استخلاص الميزات فهي عملية تكوين مجموعة جديدة من الميزات،

واستخلاص الميزات هذا يمكن أن يكون عملية مزج (تركيب) خطية أو غير خطية للميزات الأصلية [11].

\section{Recognition}

4.1

عند إدخال صورة وجه غير معروف إلى مُميّز الوجوه Face Recognizer، يتم مقارنتها مع مجموعة من الوجوه المعروفة التي تم تدريب المُمَيّز عليها و يتم إرجاع هوية صورة الوجه الأكثر تطابقاً (شبهاً) بالصورة 
المدخلة، وعملية المطابقة تقاس عادة باستخدام بعض المقاييس البعدية Distance Metric مثل المسافة

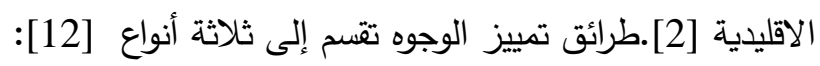

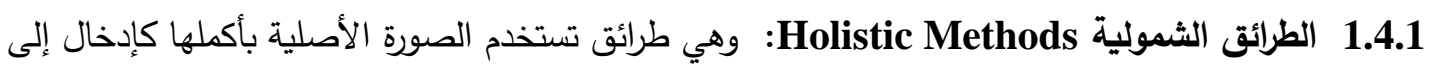
نظام تمييز الوجوه مثل PCA,LDA,ICA وغيرها. 2.4.1 الطرائق المعتمدة على الميزات Feature Based Methods: يتم في هذه الطريقة أولا إيجاد نقاط

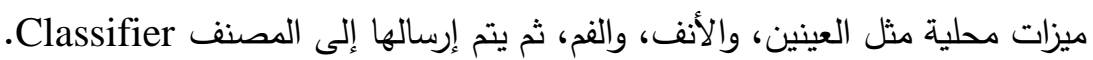
3.4.1 طرائق هجينة Hybrid Method: وهي طرائق تستخدم الميزات المحلية بالإضـافة إلى منطقة الوجه كاملة في التمييز، وتعطي هذه الطرائق الهجينة أداءً أفضل من استخدام كل طريقة من الطرائق السابقة بصورة

2.

خوارزمية AdaBoost هي خوارزمية جديدة نسبيا اقترحها العالمان Freund و و وهي منحدرة من خوارزمية الأغلبية المرجحة The Weighted Majority Algorithm المقدمة من قبل

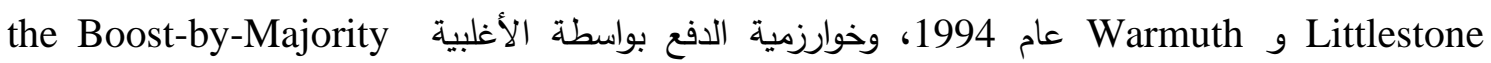
Algorithm Classifiers تعتمد خوارزمية AdaBoost على ثلاث أساسيات مهمة وهي [14]: تمثيل جديد للصورة يسى بالصورة المتكاملة (التامة) Integral Image والذي يسمح للميزات المستخدمة من قبل الكاشف أن تُحسب بسرعة عالية، إذ لا يعمل نظام الكثف هذا بصورة مباشرة مع نقاط الصورة وإنما

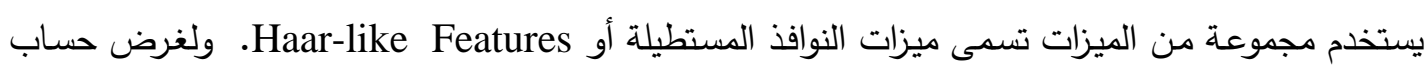
هذه الميزات بسرعة عالية وبأحجام عديدة At Many Scales تم تقديم هذا التشثيل الجديد للصورة والذي يمكن حسابه من الصورة الأصلية عن طريق مجموعة عمليات تجرى لكل نقطة فيها، وعند حساب الصورة المتكاملة فان عملية حساب أي من الميزات Haar-like Feature وعند أي موقع وبأي حجم تتم بزمن ثابت .Constant Time يتم بناء مصنف بسيط وفعال عن طريق اختيار عدد قليل من الميزات المهمة من مجموعة كبيرة من الميزات المحتملة باستخدام خوارزمية التدريب AdaBoost، داخل أي نافذة جزئية Sub Window في الصورة فان مئن العدد الكلي لميزات الـ Haar-like يكون كبيراً جداً، ولغرض ضمان عملية تصنيف سريعة فان عملية التدريب يجب ان تستبعد أغلبية الميزات المتوفرة وتركز على مجموعة صغيرة من الميزات الحساسة. ينجز اختيار الميزة باستخدام خوارزمية التدريب AdaBoost Learning Algorithm عن طريق جعل كل مصنف ضعيف يعتمد فقط على ميزة واحدة، ونتيجة لذلك فان كل مرحلة من عملية الدفع Boosting Process يتم اختيار مصنف ضعيف جديد وتظهر هذه العملية كأنها عملية اختيار ميزة جديدة. 
> ربط مجموعة المصنفات الفعالة في سلسلة Cascade Structure والذي يعمل على زيادة سرعة الكاشف بصورة ديناميكية، إذ انه يعمل على إهمال مناطق الصورة التي لا تحتوي وجه (الخلفية) بالمراحل الأولى من السلسلة وتبقى المناطق المحتمل وجود الوجه فيها لتدخل معالجات إضافية خلال المراحل التالية من السلسلة.

\section{Features}

تصنف الصور في خوارزمية AdaBoost بالاعتماد على قيمة ميزات بسيطة. وان سبب استخدام الميزات

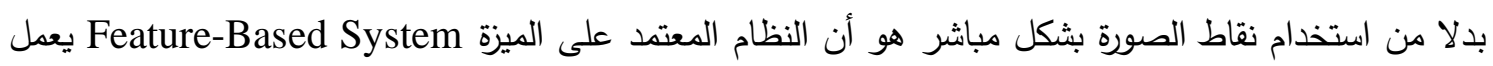

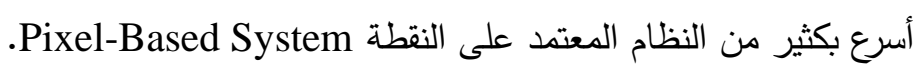

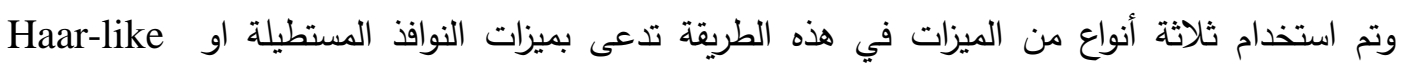

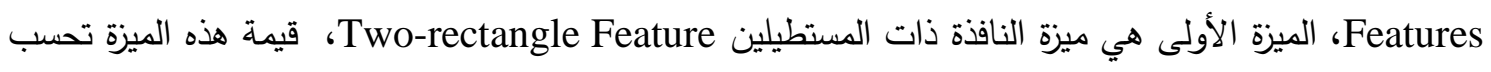
من خلال الفرق بين مجموعي النقاط داخل منطقتي المستطيلين. أما الميزة الثانية فهي ميزة النافذة ذات الثلاثة مستطيلات Three-rectangle Feature ويتم حساب قيمتها عن طريق حساب مجموع النقاط داخل المستطيلين الخارجيين ويتم طرحهم من مجموع النقاط للمستطيل الوسطي. أما الميزة الثالثة فهي ميزة النافذة ذات الثاب الأربعة مستطيلات Four-rectangle Feature وتحسب قيمتها من خلال حساب الفرق بين مجموع نقاط الأزواج القطرية للمستطيلات. علماً ان مناطق المستطيلات في كل ميزة لها نفس الحجم والثكل، وأما تكون متجاورة بثكل أفقي أو

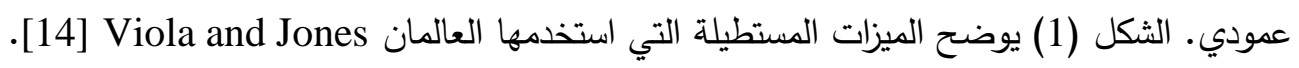

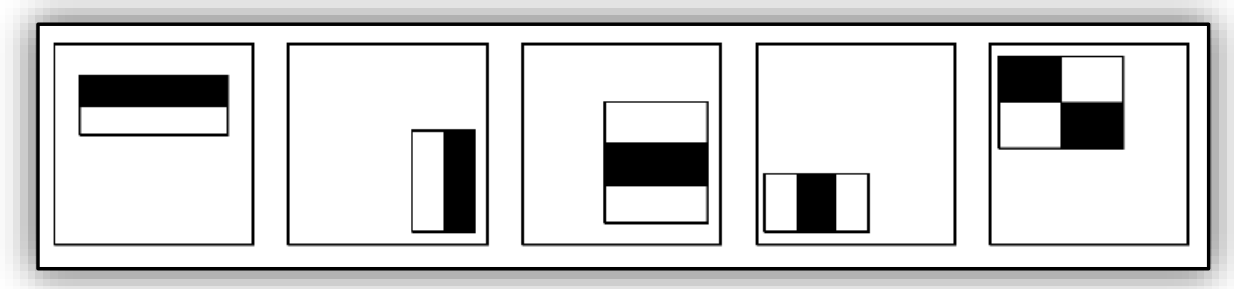

\section{Viola and Jones الثكل(1) نوافذ الميزات المستطيلة المستخدمة من قبل العالمان}

\section{Integral Image}

2.2

إن الميزات المستطيلة يمكن أن تُحسب بطريقة سريعة باستخدام تمثيل وسطي جديد للصورة يسمى الصورة

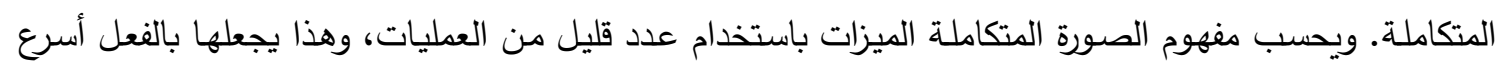
مقارنة مع غيرها من الخوارزميات.

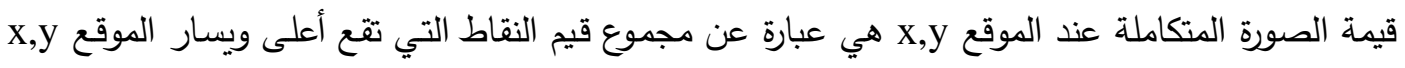

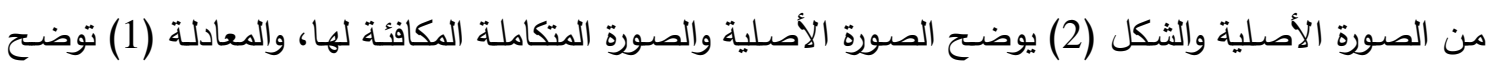

$$
i i(x, y)=\sum_{x^{\prime} \leq x, y^{\prime} \leq y} i\left(x^{\prime}, y^{\prime}\right)
$$


و باستخدام المعادلتين (2) و(3) وبصورة متكررة لكل نقطة فان الصورة المتكاملة يمكن أن تحسب بخطوات ابسط

$\mathrm{s}(\mathrm{x}, \mathrm{y})=\mathrm{s}(\mathrm{x}, \mathrm{y}-1)+\mathrm{i}(\mathrm{x}, \mathrm{y})$

واقصر على الصورة الأصلية: بالمان

$\mathrm{ii}(\mathrm{x}, \mathrm{y})=\mathrm{ii}(\mathrm{x}-1, \mathrm{y})+\mathrm{s}(\mathrm{x}, \mathrm{y})$

إذ أن s(x,y) يمثل مجموع السطر التراكمي s(x,-1)=0 The cumulative row sum ،

\begin{tabular}{|l|l|l|l|l|}
\hline 3 & 2 & 5 & 6 & 1 \\
\hline 3 & 4 & 5 & 5 & 6 \\
\hline 3 & 3 & 5 & 3 & 2 \\
\hline 2 & 4 & 4 & 4 & 3 \\
\hline 3 & 2 & 2 & 4 & 2 \\
\hline
\end{tabular}

الصورة ألأصنية

\begin{tabular}{|r|r|r|r|r|}
\hline 3 & $\mathbf{5}$ & $\mathbf{1 0}$ & $\mathbf{1 6}$ & $\mathbf{1 7}$ \\
\hline 6 & $\mathbf{1 2}$ & $\mathbf{2 2}$ & $\mathbf{3 3}$ & $\mathbf{4 0}$ \\
\hline $\mathbf{9}$ & $\mathbf{1 8}$ & $\mathbf{3 3}$ & $\mathbf{4 7}$ & $\mathbf{5 6}$ \\
\hline 11 & $\mathbf{2 4}$ & $\mathbf{4 3}$ & $\mathbf{6 1}$ & $\mathbf{7 3}$ \\
\hline $\mathbf{1 4}$ & $\mathbf{2 9}$ & $\mathbf{5 0}$ & $\mathbf{7 2}$ & $\mathbf{8 6}$ \\
\hline
\end{tabular}

الصورة المتكامدة

\section{الثكل (2) الصورة الأصلية والصورة المتكاملة المكافئة لها}

Four وباستخدام الصورة المتكاملـة فـان مجموع أي مستطيل يمكن أن يحسب باستخدام أربع نقاط استـلال References Points، والثكل (3) يوضح طريقة حساب مجموع نقاط المستطيل من خلال الصورة المتكاملة.

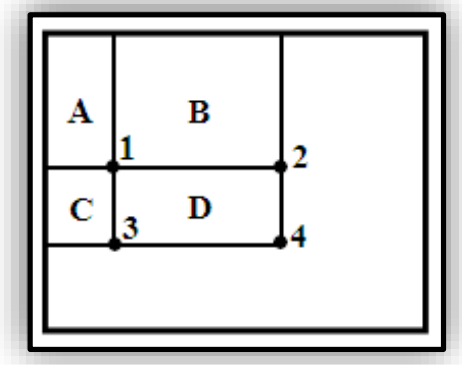

\section{الثكل (3) طريقة حساب مجموع نقاط المستطيل من خلال الصورة المتكاملة}

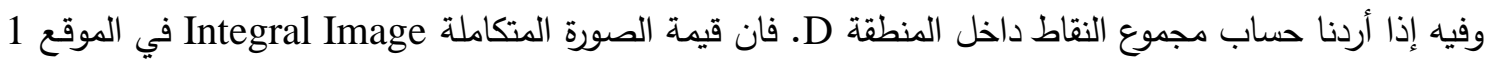
هو مجموع النقاط في المستطيل A. والقيمة في موقع 2 هو A+B، وفي موقع 3 هو A+C، وعند موقع 4 هو D + C + A + B

أما بالنسبة للميزات المستخدمة في تقنية كثف الوجه والتي تتكون من مستطيلات متجاورة، فلحساب قيمة فئس الميزة ذات المستطيلين نحتاج إلى ست نقاط استدلال، وقيمة الميزة ذات الثلاثة مستطيلات تحتاج إلى ثمان نقاط

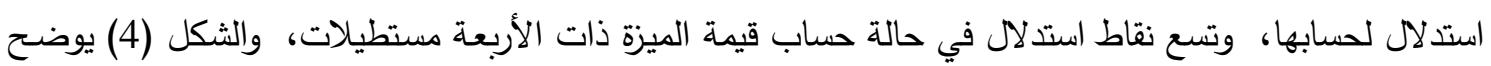
عدد المصادر لحساب كل منها[114]. 


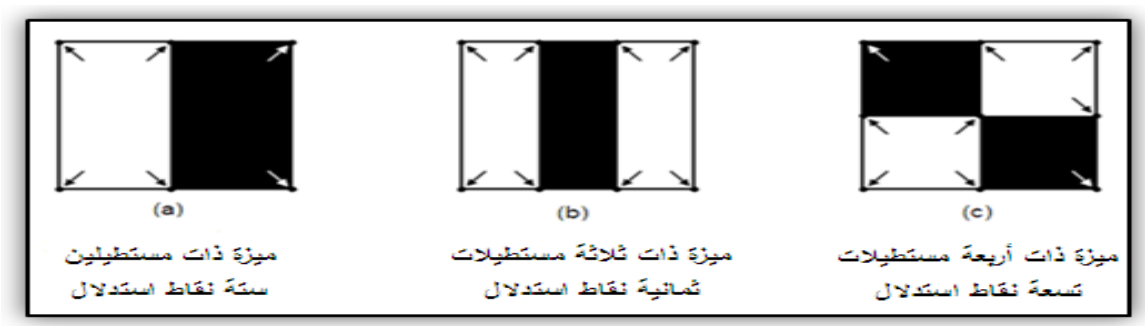

الثكل (4) عدد نقاط الاستدلال لحساب قيمة كل نوع من الميزات المستطيلة، الزوايا المؤشرة بالأسهم تمثل نقاط الاستدلال التي تحتاجها الطريقة لحساب الميزة

AdaBoost 3.2

إن استخدام الميزات المستطيلة بأحجام ومواقع مختلفة من نافذة البحث يؤدي إلى توليد عدد كبير جدا من

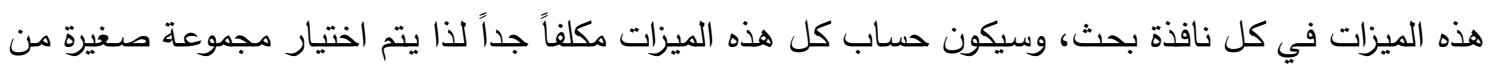
هذه الميزات لتثكيل المصنف الفعال (القوي) عن طريق دمج خطي لتلك الميزات المختارة وذلك باستخدام خوارزمية التدريب AdaBoost [15] فيزه

تستقبل خوارزمية التدريب AdaBoost مجموعة من نماذج مجاميع التدريب Training-Set بعضها يحوي وجه وبعضها الآخر لا يحوي وجه وهذه النماذج تكون مؤشرة Labeled إذ يتم إعطاء (0) لأنموذج التدريب الذي نمادي

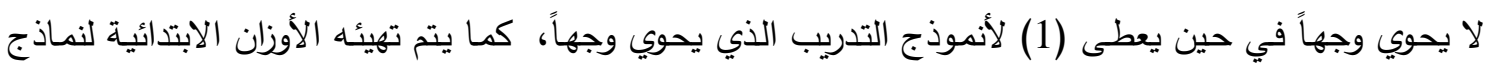

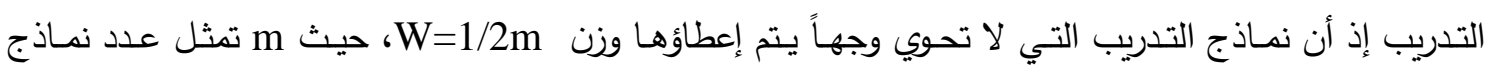

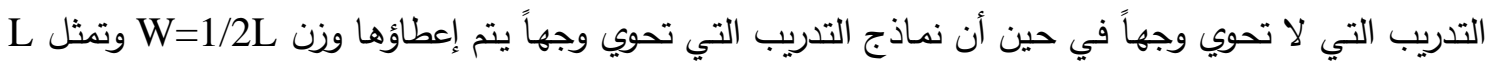
عدد نماذج التدريب التي تحوي صورة وجه. كما تستقبل خوارزمية التدريب AdaBoost مجموعة المصنفات الضعيفة (ميزة مستطيلة/ حد عتبة)، فإذا كان عدد نماذج صور التدريب الكلي N، وعدد الميزات المستطيلة المستخدمة K، فان عدد المصنفات الضعيفة سيكون مساوياً لـ KN، حيث أن كل ميزة مستطيلة واحدة لها حد عتبة مختلف مع كل صورة من صور التدريب بحيث تصنف تلك الصورة إلى وجه في حالة احتوائها على صورة وجه أو إلى لا يوجد وجه في حالة عدم احتوائها

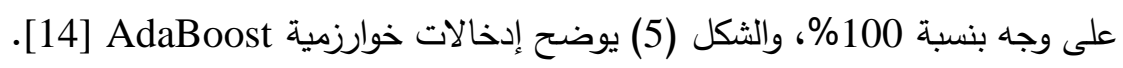

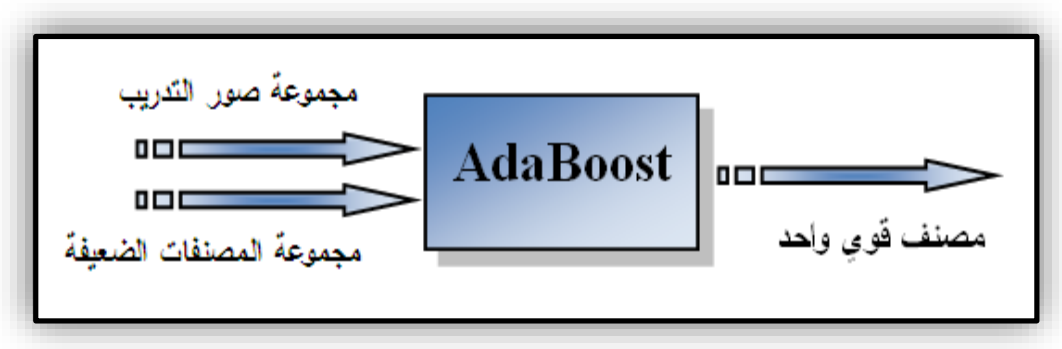

\section{AdaBoost الثكل (5) ادخالات خوارزمية}

يتم في كل دورة اختيار مصنف ضعيف ذو اقل نسبة خطأ من بين عدد كبير من المصنفات الضعيفة،

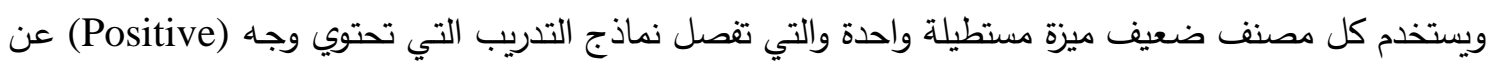

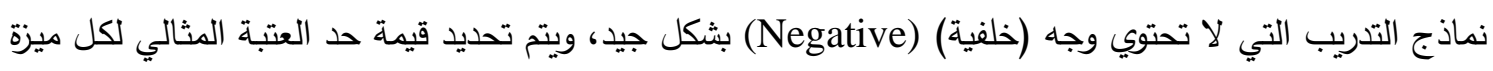


والتي عن طريقها لا يتم تصنيف عدد قليل فقط من نماذج التدريب بشكل صحيح، وهذه العملية تكرر في كل دورة. والمصنف النهائي والذي يسمى المصنف القوي Strong Classifier يُكوّن من خلال عملية دمج خطية موزونة تصنة للمصنفات الضعيفة التي تم اختيارها [15]. والثكل (6) يبين أول ميزتين تم اختيارهم من قبل خوارزمية التدريب لكني AdaBoost

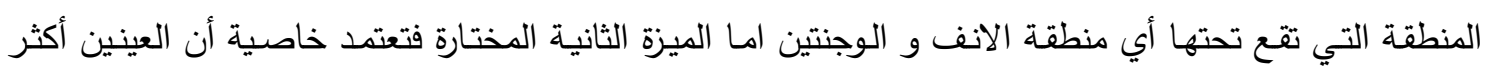

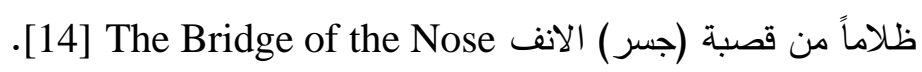

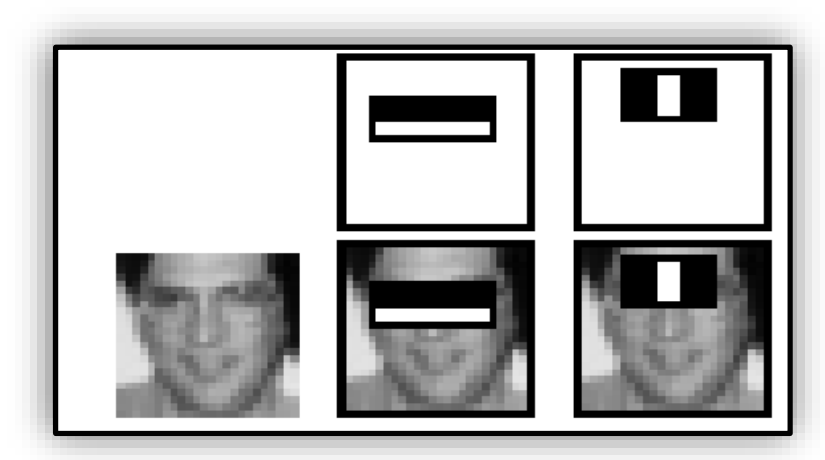

الثكل (6) اول ميزتين تم اختيارهم من قبل خوارزمية التدريب AdaBoost عند تطبيقها من قبل العالمان Viola and Jones

\section{Cascade of Classifiers}

4.2 سلسلة المصنفات

تحتوي كل صورة يتم معالجتها عدداً كبيراً من نوافذ البحث والتي يتم اختبارها الواحدة تلو الأخرى لكثف

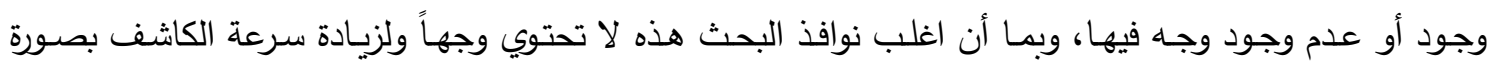

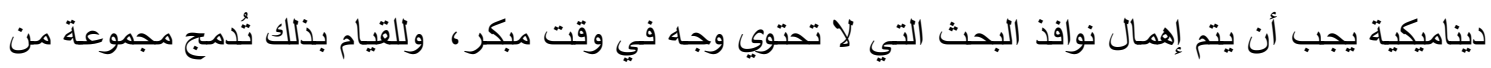

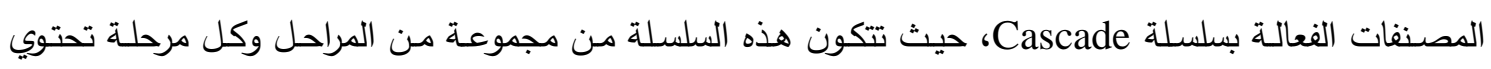
مصنف فعال لتصنيف نوافذ البحث إما إلى وجه أو لا يوجد وجه. تحتوي المراحل الأولى من السلسلة على عدد قليل من المصنفات الضعيفة وتُقتيّم فيها نوافذ البحث بصورة

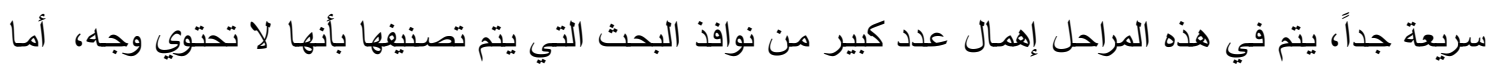

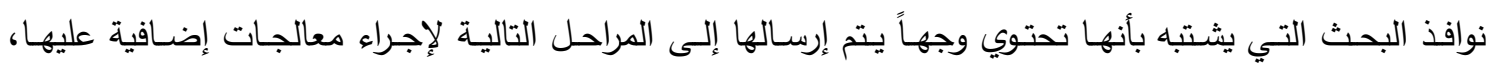

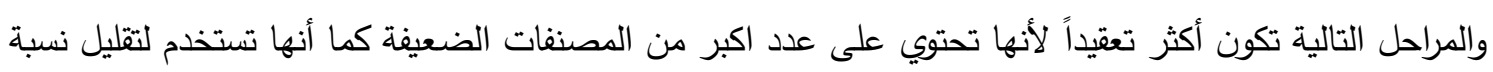
الكثف الخاطئ. وهكذا فان جزء الصورة داخل نافذة البحث يصنف كوجه Face إذا تم تصنيف نافذة البحث هذه في كل

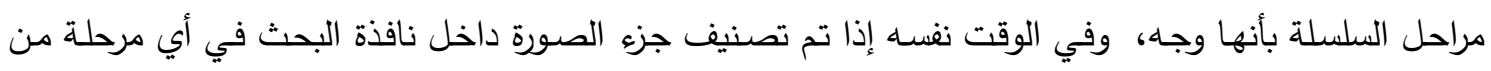

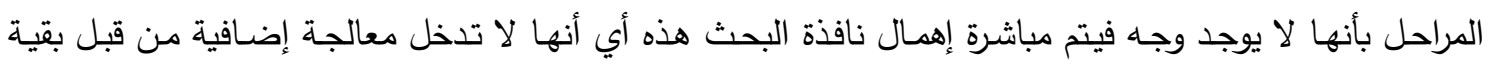

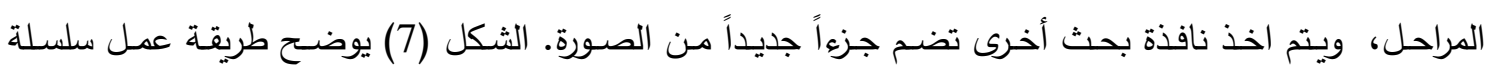




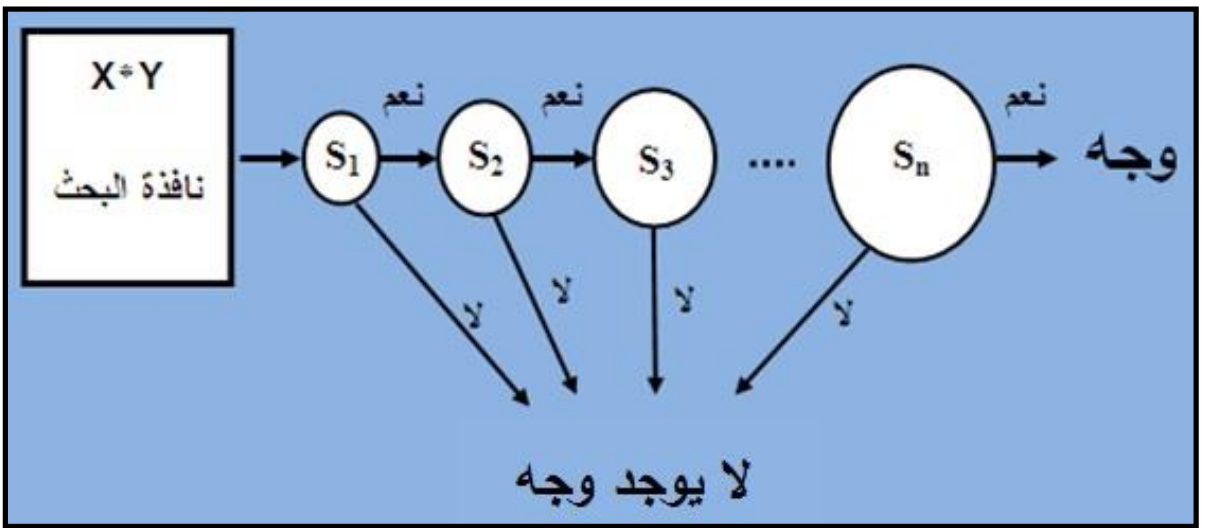

الثكل (7) طريقة عمل سلسلة المصنفات الفعالة

Principle Component Analysis (PCA)

3. تحليل المركبات الأساسية

تحليل المركبات الأساسية هي تقنيـة قياسية تستخدم في تمييز الأنماط و معالجـة الإثـارة، وهي طريقـة

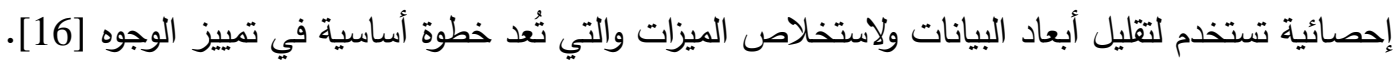

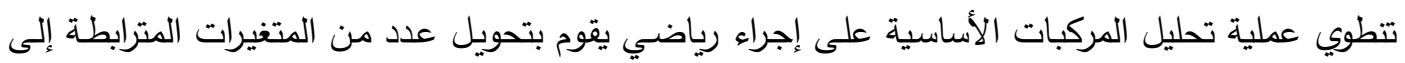
عدد من المتغيرات غير المترابطة تسمى بالمركبات الأساسية، وترتبط هذه المركبات بالمتغيرات الأصلية عن طريق لهني

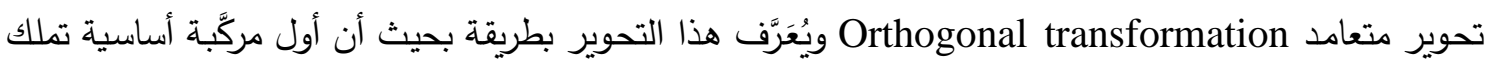
أعلى تباين وثاني مركَّبة أساسية تملك ثاني أعلى تباين وهكذا. لـا PCA تسميات عديدة بالاعتماد على ميدان تطبيقها منها:

Karhunen-Loe've Transform (KLT), The Hotelling Transform or Proper Orthogonal Decomposition.

يتم رياضياً إيجاد المركبات الأساسية لتوزيع الوجوه باستخدام طريقة Eigen Face إذ يتم أولا إيجاد متجهات الايكن Eigen Vectors لمصفوفة التباين لمجموعة من صور الوجوه المستخدمة في التدريب ثم يتم ترتيبها تتازليا حسب قيم الايكن لها Eigen Values، ومن ثم تحديد قيمة معينة كحد عتبة حيث يتم اهمال متجهات الايكن التي تكون قيم الايكن لها اقل من حد العتبة، بعد ذلك يتم تخطيط صور الوجوه الى مجموعة متجهات الايكن التي تم اختيارها للحصول على مجموعة تسمى بـ Eigen Faces والتي تعد مجموعة من الميزات وتوصف معاً الاختلاف Eigen بين صور الوجوه المستخدمة في التدريب, حيث أن كل صورة وجها تسهم بمقدار معين في تكوين الـ Eئل كما أن عددها مساوياً لعدد صور الوجوه المستخدمة في التدريب. Faces الفائدة الرئيسية من الـ PCA هي أن طريقة الـ Eigen Face تساعد بتقليل حجم قاعدة البيانات المطلوبة لتمييز صورة الوجه المدخلة في عملية الاختبار • إذ لا يتم خزن الصور المستخدمة في التدريب مباشرة وإنما يتم

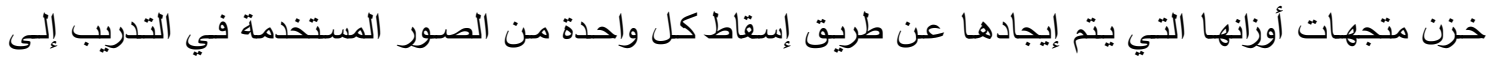
مجموعة من ال. Eigen Faces التي تم حسابها. ويتم اسقاط صورة الوجه المدخلة في الاختبار إلى مجموعة الـ Eigen Faces للحصول على أفضل تطابق[17].

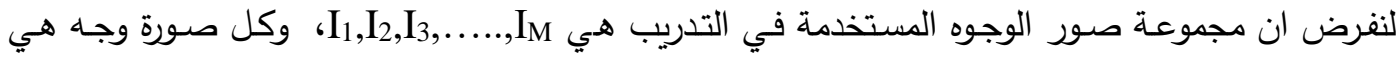
عبارة عن مصفوفة ثنائية البعد (n*m) فانه [18]: 
يتم تحويل كل صورة وجه في مجموعة التدريب الى متجه Vector ذو بعد (nm*1).

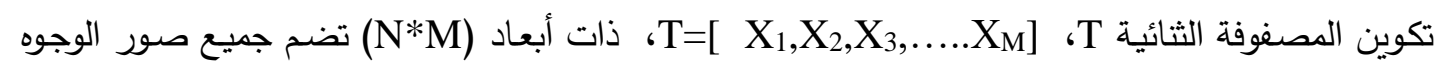
المستخدمة في التدريب، عدد الأعمدة في المصفوفة T مساويا لعدد صور الوجوه (M) وكل عمود X

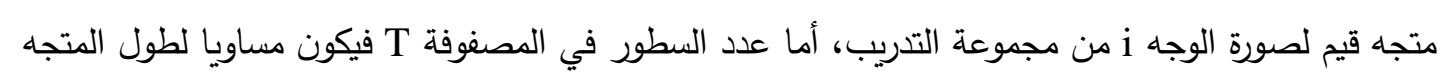
أي أن N=n*m " حساب متجه الوجه المعدل (الصورة التي تمثل المعدل لجميع صور الوجوه الدستخدة في التدريب) والذي يكون أيضا ذا بعد (nm*1) من خلال المعادلة (4) التهول $\mu=\frac{1}{M} \sum_{i=1}^{M} X_{i}$

طرح متجه الوجه المعدل من كل متجه من متجهات صور الوجوه في الموجودة في المصفوفة T بتطبيق

المعادلة (5)

$P_{i}=X_{i}-\mu \quad, i=1,2, \ldots, M$

الناتج من هذه العملية ستكون المصفوفة الثنائية A ذات بعد (N*M) وكل عمود i فيها يمثل المتجه i الناتج من الخطوة السابقة،

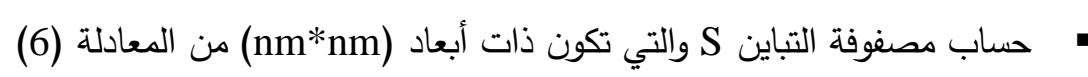

$\mathrm{S}=\mathrm{A} \cdot \mathrm{A}^{\mathrm{T}}$

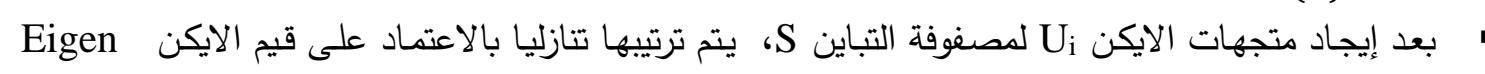

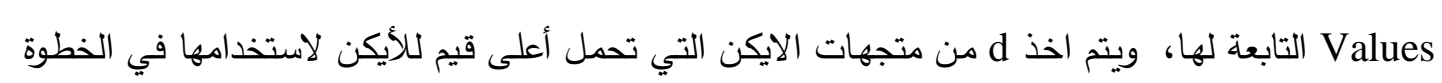

الآتية.

يتم إيجاد متجهات أوزان كل صورة وجه مستخدمة في التدريب عن طريق إسقاط كل منها على مجموعة

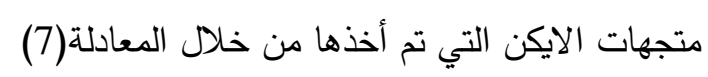

$\mathrm{W}_{\mathrm{i}}=\mathrm{U}_{\mathrm{i}}^{\mathrm{T}} * \mathrm{P}$

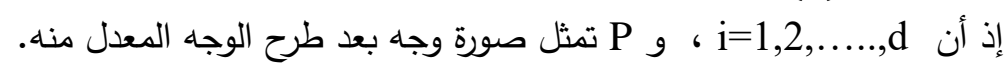

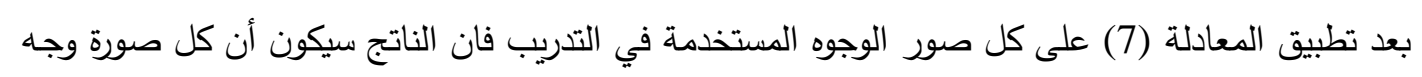

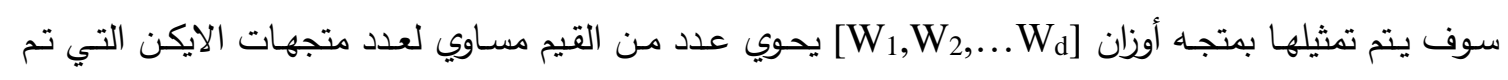
اختيارها (d) والتي سيتم خزنها في قاعدة البيانات لاستخدامها فيما بعد في عملية التصنيف.

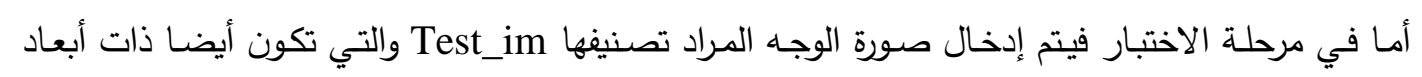

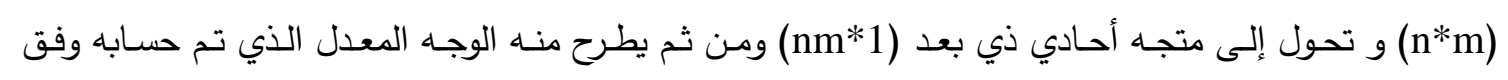

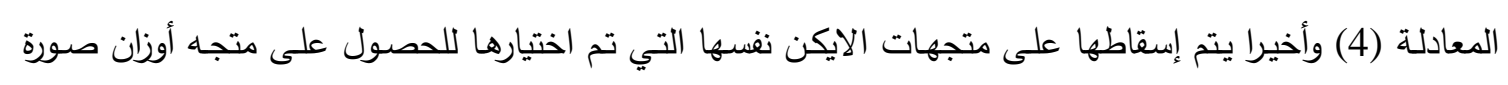
الوجه التي يتم اختبارها من خلال المعادلة (7). 


\section{Linear Discriminate Analysis(LDA)}

4. تحليل التمييز الخطي

هي طريقة تقليدية في تمييز الوجوه وهي طريقة فعالة في عملية التصنيف لأنها من الطرائق الخطية للتقليل

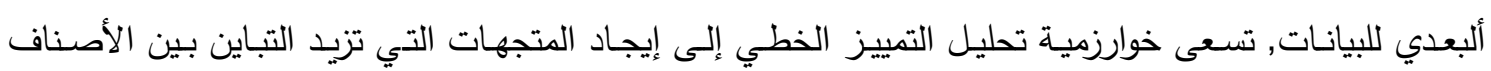

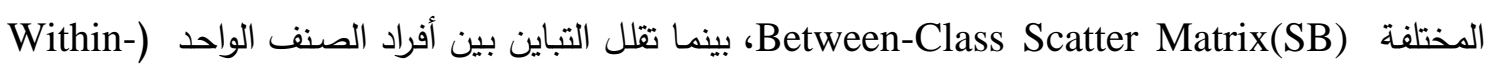
Class Scatter Matrix(SW للأصناف المختلفة. Within-Class Scatter Matrix (SW) وتسمى أيضا Intra-Personal والتي تمثل Between-Class التباينات في الظهور للثخص نفسه والناتجة بسبب اختلاف الإضاءة أو التعابير الوجهية بينما Scatter Matrix(SB) (الثخص)، والثكل (8) يوضح الفصل الجيد بين الأصناف المختلفة وداخل الصنف الواحد.

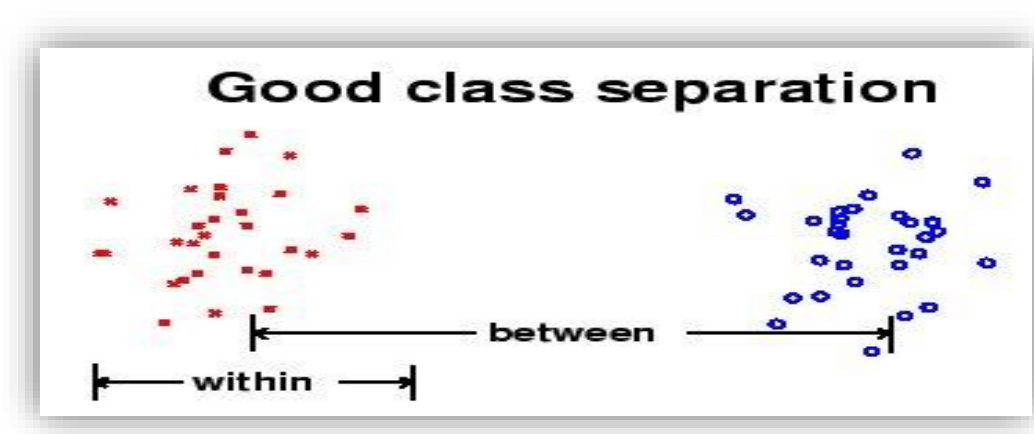

الثكل (8) يبين الفصل الجيد بين الأصناف المختلفة وداخل الصنف الواحد

ونتيجة للا LDA يتم تكوين متجه أوزان لكل صورة وجه سواء كان ضمن مجموعة التدريب أو لصورة الوجه الجديدة

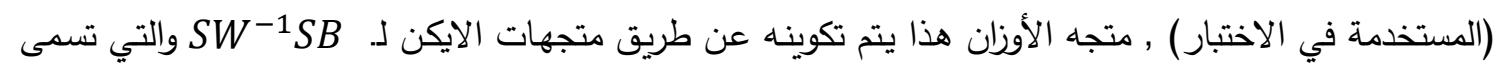
بالـ [19] Fisher Faces

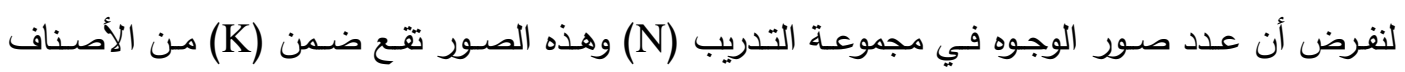

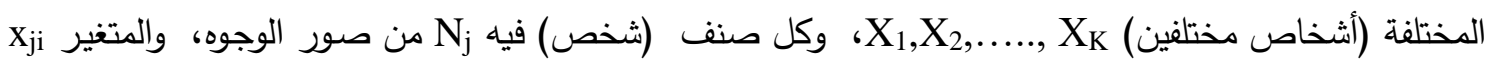

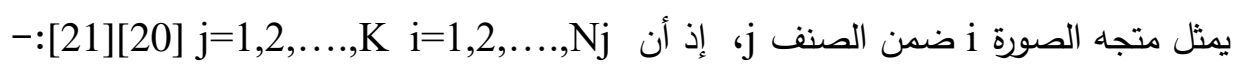
يتم تحويل صور الوجوه المستخدمة في التدريب من مصفوفات ثنائية الى متجهات أحادية.

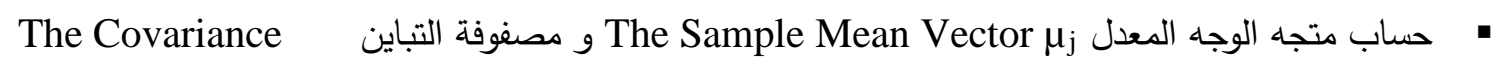
Matrix $S_{j}$ $\mu_{\mathrm{j}}=\frac{1}{\mathrm{~N}_{\mathrm{j}}} \sum_{\mathrm{i}=1}^{\mathrm{N}_{\mathrm{j}}} \mathrm{x}_{\mathrm{ji}}$

$S_{j}=\frac{1}{N_{j}} \sum_{i=1}^{N_{j}}\left(x_{j i}-\mu_{j}\right)\left(x_{j i}-\mu_{j}\right)^{T}$

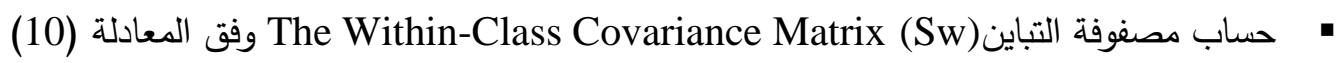


$\mathrm{Sw}=\sum_{\mathrm{j}=1}^{\mathrm{K}} \mathrm{P}\left(\mathrm{K}_{\mathrm{j}}\right) \mathrm{S}_{\mathrm{j}}$

إذ أن:

The Prior Class Probability j ويمثل احتمالية سابقة للصنف p(K $\left.K_{j}\right)=1 / K$ Kمثل عدد الأصناف (الأشخاص) K

• يتم حساب إجمالي الوجه المعدل The Overall Mean $\mu$ وفق المعادلة (11)

$\mu=\frac{1}{N} \sum_{\mathrm{j}=1}^{\mathrm{K}} \sum_{\mathrm{i}=1}^{\mathrm{N}_{\mathrm{j}}} \mathrm{x}_{\mathrm{ji}}=\frac{1}{\mathrm{~N}} \sum_{\mathrm{j}=1}^{\mathrm{K}} \mathrm{N}_{\mathrm{j}} \mu_{\mathrm{j}}$

إذ أن N يمثل عدد الصور الكلي في مجموعة التدريب

• حساب مصفوفة التباين The Between-Class Covariance Matrix SB من خلال المعادلة (12) $\mathrm{SB}=\sum_{\mathrm{j}=1}^{\mathrm{K}} \mathrm{P}\left(\mathrm{K}_{\mathrm{j}}\right)\left(\mu_{\mathrm{j}}-\mu\right)\left(\mu_{\mathrm{j}}-\mu\right)^{\mathrm{T}}$

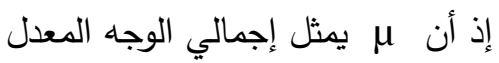

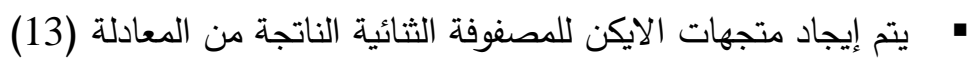

$\mathrm{W}=\frac{\mathrm{SB}}{\mathrm{SW}}$

• حساب متجه الأوزان لكل صورة وجه ضمن مجموعة التدريب ولصور الوجوه المستخدمة في الاختبار وفق

Training_Vector $=\mathrm{W}^{\mathrm{T}}$.Image_Train_Vector

(15) المعادلات (14) و (14) (15)

Test_Vector $=\mathrm{W}^{\mathrm{T}}$.Image_Test_Vector

\section{Back Propagation Network}

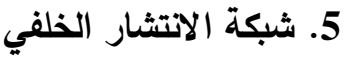

وهي شبكة تعلم بإشراف [22]، تم اكتثافها من قبل عدد من الباحثين وهم (Hinton ,Williams) في

العام 1986 و (Rumehart McClellands, في العام 1988 ولقد لعبت دوراً رئيساً في إعادة بروز الثبكات العصبية الاصطناعية بوصفها أداةً لحل الكثير من المشاكل على نطاق واسع [23].

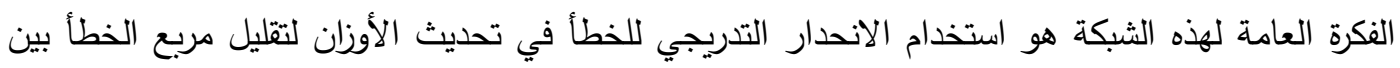
قيم الاخراجات للثبكة وقيم اخراجات الهدف، إذ يتم استخدام مقدار الخطأ الناتج بقوانين التحديث لتغيير الأوزان عند كل طبقة Layer من الثبكة ابتداءً من طبقة الإخراج وانتهاءً بطبقة الإدخال ولذلك سميت بشبكة الانتثار الخلفي،وقد تم اختيارها مصنفاً في النظام المقترح بسبب بساطتها وقدرتها في التعرف على نمط تحت إشراف [22]. 5.1 خوارزمية تعليم شبكة الانتشار الخلفي إن عملية تعليم (تدريب) شبكة الانتشار الخلفي تمر بالمراحل التالية [24]: 


\section{Forward Propagation}

أ. مرحلة الاتتشار الأمامي

تنتشر الإشارة الداخلة من طبقة الإدخال Input Layer خلال الطبقة (الطبقات) المخفية وصولا إلى طبقة الإخراج Output Layer. خلا مرحلة الانتثار الأمامي من معالجة الإثارة فان قيمة الوزن وقيمة عامل

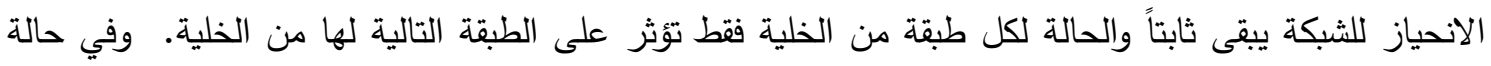
عدم تحقيق الإخراج المطلوب Target عند طبقة الإخراج يتم التحويل إلى الانتشار الخلفي لنشر إثارة الخطأ

.The Error Signal

\section{Back Propagation}

ب. مرحلة الاتتشار الخلفي (التغذية العكسية)

يدعى الفرق بين الإخراج الحقيقي للشبكة والإخراج المطلوب (Target) بـ إشارة الخطأ Error Signal. تتشر في هذه المرحلة إثارة الخطأ من طبقة الإخراج إلى طبقة الإدخال طبقة بعد أخرى، كما تُعدّل قيمة الأوزان

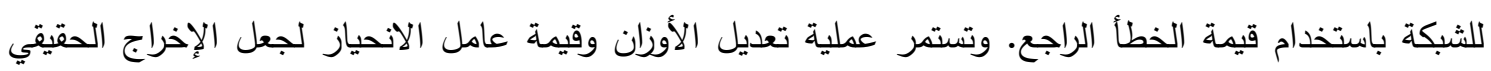
للشبكة مساوياً تقريباً للإخراج المطلوب.

\section{The Proposed System}

6. النظام المقترح

إن فكرة عمل النظام المقترح هو تمييز الأشخاص ومعرفة هويتهم في حالة وجودهم في قاعدة بيانات النظام (مدرّب عليهم) فضلاً عن تمييز الأشخاص الذين لا يملكون صوراً لهم في قاعدة البيانات (غير المدرّب عليهم)

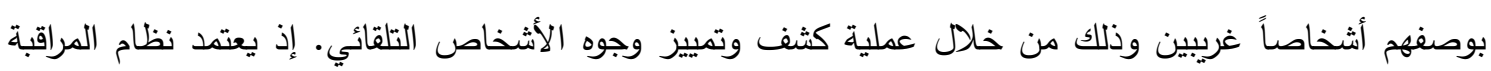
المقترح على تدفق فيديوي حي live video يتم الحصول عليه من كاميرا مراقبة.

$$
\text { يتكون النظام من أربع مراحل رئيسة: }
$$

1. مرحلة تكوين قاعدة البيانات حيث تتم بصورة آنية. فعند تفعيل هذه المرحلة يتم الحصول على تدفق فيديوي حي

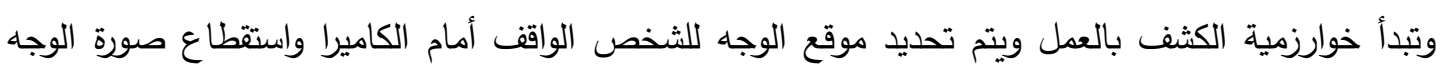
وخزنها فضلاً عن معلومات أخرى مثل اسم الثخص وعمره ورقم الصورة المأخوذة. 2. مرحلة استخلاص الميزات ويتم فيها تحميل الصور التي تم أخذها للأثخاص وتطبيق خوارزمية تحليل المركبات

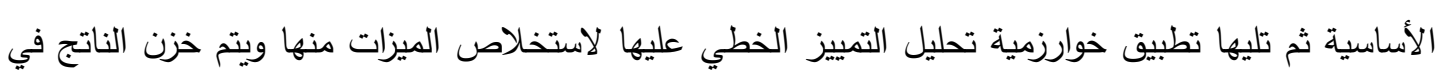

$$
\text { ملفات نصية. }
$$

3. مرحلة تدريب الثبكة العصبية على الميزات التي تم استخلاصها من صور الوجوه.

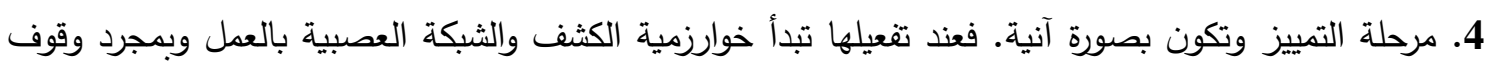

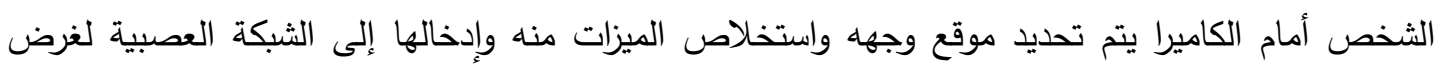
استرجاع معلوماته المخزنة والمتمثلة باسم الشخص وعمره. والثكل (9) يوضح مخطط ولثن والنظام المقترح.

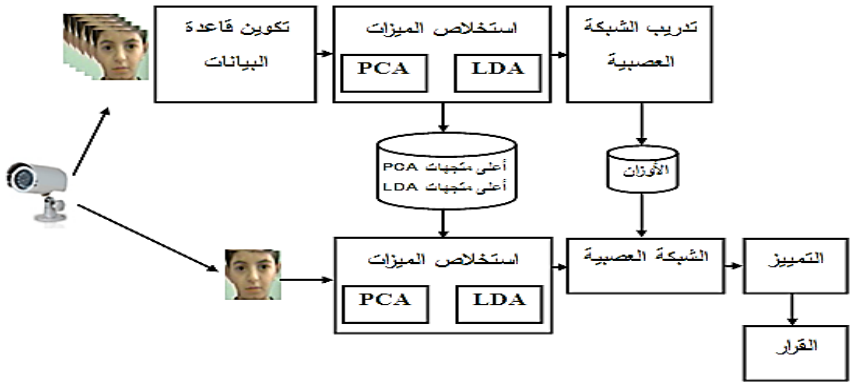

الشكل (9) مخطط النظام المقترح 
تم إجراء العديد من التجارب لبيان نتائج تطبيق النظام المقترح، وقد تم في البداية إجراء التجربة الاولى على التدفق الفيديوي الحي وفيها تم حساب نسبة التمييز بالاعتماد على Frame 12 من كل ثانية على اعتبار انها

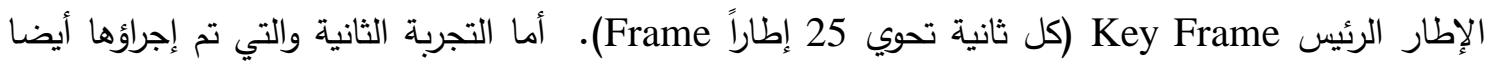

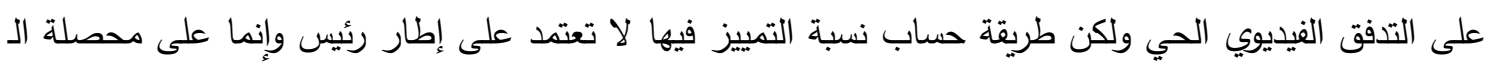
25 إطاراً. أي يتم اخذ اسم الثخص الأكثر تكراراً خلال الـ 25 إطاراً.

1.7 التجربة الاولى:

تم تطبيق طريقة التمييز المقترحة بصورة آنية على التدفق الفيديوي الحي، إذ تم اخذ صور لمجموعة من الأشخاص البالغ عددهم (10) شخص، وتم اخذ (40) صورة لكل منهم وخزنها لتكوين قاعدة البيانات وتمت عملية اخذ الصور بصورة آنية وبكاميرا المراقبة المستخدمة في التمييز نفسها، وتم وتم في هذه التجربة الاعتماد على الاطار الرئيس في حساب نسبة التمييز ·

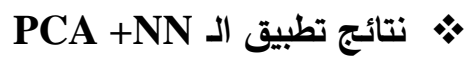

تم حساب نسب التمييز في حالة استخدام طريقة تحليل المركبات الأساسية وحدها في استخلاص الخواص والثبكة العصبية في التمييز وبالاعتماد على الإطار الرئيس، فعند اخذ 100 من متجهات الايكن ذات الاعلى Eigen Values 83.9\% وانخفضت هذه النسبة الى 80.3\% ثم الى 79.4\% عندما تم اخذ 200 و250 على التوالي من متجهات

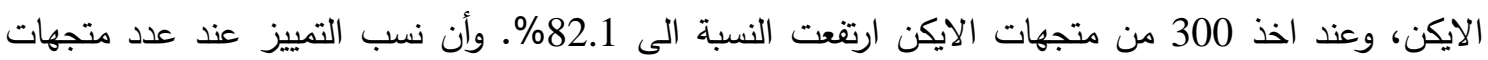

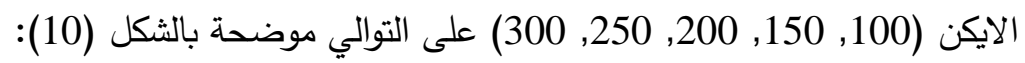

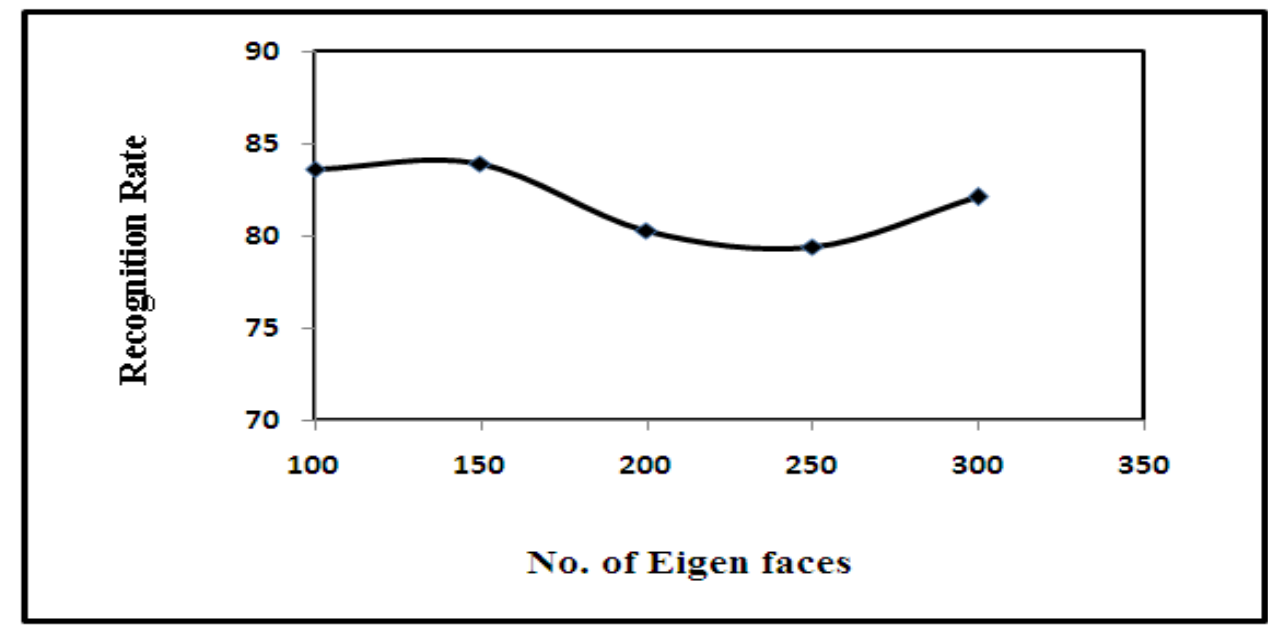

الثكل (10) نسب التمييز الآني لطريقة PCA+NN في التجربة الاولى

PCA +LDA+NN نتائج تطبيق الـ

تم حساب نسبة التمييز في حالة اختيار (300,250,200,150,100) على التوالي من متجهات الايكن في الـ PCA، ولكل حالة تم اختيار قيم معينة من متجهات الايكن في الـ LDA وتم حساب نسبة التمييز في كل حالة

وتوضيحها بالجدول (1). 
جدول (1) نتائج التمييز الآني لطريقة PCA+LDA+NN في التجربة الاولى

\begin{tabular}{|c|c|c|c|c|c|}
\hline PCA & LDA & نسبة القبول & نسبة الرفض & وجه مكان آخر & نسبة التمييز \\
\hline \multirow{4}{*}{100} & 25 & 9.0312 & 8.0313 & 2.125 & 80.8125 \\
\hline & 50 & 5.4687 & 6.25 & 0.7812 & 87.5 \\
\hline & 75 & 7.0312 & 4.6875 & 0.7812 & 87.5 \\
\hline & 100 & 8.5937 & 4.6875 & 0.7812 & 85.9375 \\
\hline \multirow{3}{*}{150} & 50 & 11.7647 & 3.6764 & 1.4705 & 83.0882 \\
\hline & 100 & 8.5937 & 2.3437 & 2.3437 & 86.7187 \\
\hline & 150 & 11.4503 & 1.5267 & 0.0 & 87.0229 \\
\hline \multirow{4}{*}{200} & 50 & 7.7521 & 3.5503 & 3.8759 & 84.8217 \\
\hline & 100 & 8.5938 & 5.3437 & 3.125 & 82.9375 \\
\hline & 150 & 7.7521 & 7.6511 & 2.3255 & 82.2713 \\
\hline & 200 & 8.5273 & 2.3255 & 3.1007 & 86.0465 \\
\hline \multirow{5}{*}{250} & 50 & 10.8921 & 3.7037 & 1.8601 & 83.5441 \\
\hline & 100 & 9.7014 & 2.9850 & 2.2388 & 85.0746 \\
\hline & 150 & 8.9668 & 3.6807 & 2.5316 & 84.8209 \\
\hline & 200 & 10.3705 & 5.4411 & 2.7440 & 81.4444 \\
\hline & 250 & 12.3531 & 6.1851 & 1.7581 & 79.7037 \\
\hline \multirow{6}{*}{300} & 50 & 7.4717 & 5.6511 & 4.2018 & 82.6754 \\
\hline & 100 & 8.8124 & 8.8125 & 3.3438 & 79.0313 \\
\hline & 150 & 6.5217 & 6.5217 & 2.1739 & 84.7826 \\
\hline & 200 & 8.2089 & 5.2239 & 0.0 & 86.5672 \\
\hline & 250 & 6.9767 & 5.4264 & 3.1007 & 84.4961 \\
\hline & 300 & 7.8125 & 3.9063 & 3.125 & 85.1562 \\
\hline
\end{tabular}

الثكل (11) يوضح صور الاشخاص الذين تم تمييزهم بصورة آنية في التجربة الاولى والثانية كما يوضح الثكل (12) صورة الوجه المعدّل وصور Eigen Faces ذات الأعلى قيم الايكن لمجموعة صور الوجوه المستخدمة في التدريب في التجربة الاولى والثانية.

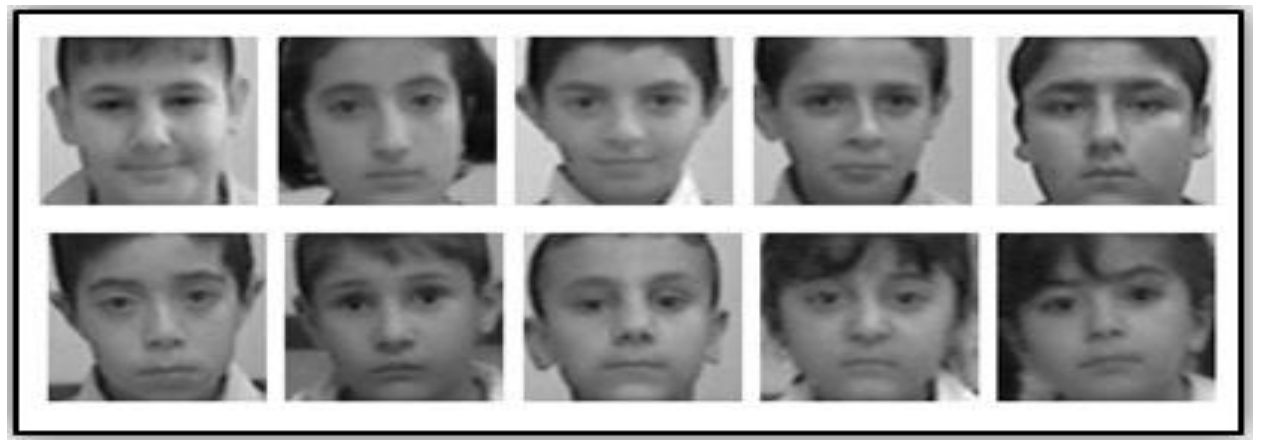

الثكل (11) صور الأشخاص الذين تم تمييزهم في التجربة الاولى والثانية 


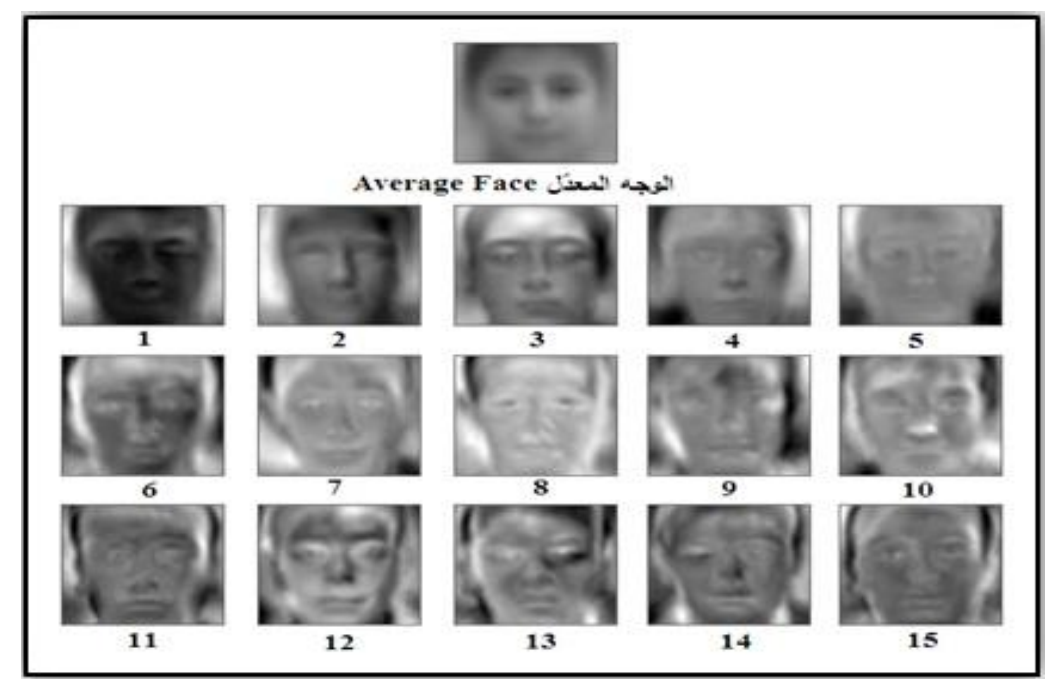

الثكل (12) صورة الوجه المعدل و 15 صورة لمتجهات الايكن ذات الأعلى قيم أيكن

2.7

هذه التجربة مشابهة للتجربة السابقة ماعدا ان طريقة حساب نسبة التمييز لا تعتمد على إطار رئيس وإنما على محصلة الـ 25 إطاراً أي يتم اخذ اسم الثخص الأكثر تكراراً خلال الـ 25 إطار .

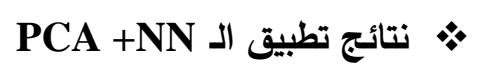

تم حساب نسب التمييز في حالة استخدام طريقة تحليل المركبات الأساسية وحدها في استخلاص الخواص

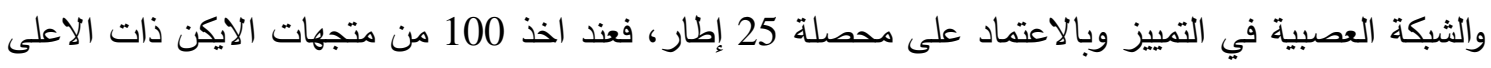
قيم الايكن كانت نسبة التمييز 86.3\%، وعند اخذ 150 من متجهات الايكن ارتفعت نسبة التمييز الى هـ 87.8\%، وانخفضت هذه النسبة الى 84.3\% ثم الى 83.6\% عندما تم اخذ 200 و250 على التوالي من متجهات الايكن، وعند اخذ 300 من متجهات الايكن ارتفعت النسبة الى 84.5\%. وأن نسب التمييز عندما تكون عدد متجهات الايكن (300,250,200,150,100) على التوالي موضحة بالثكل (13):

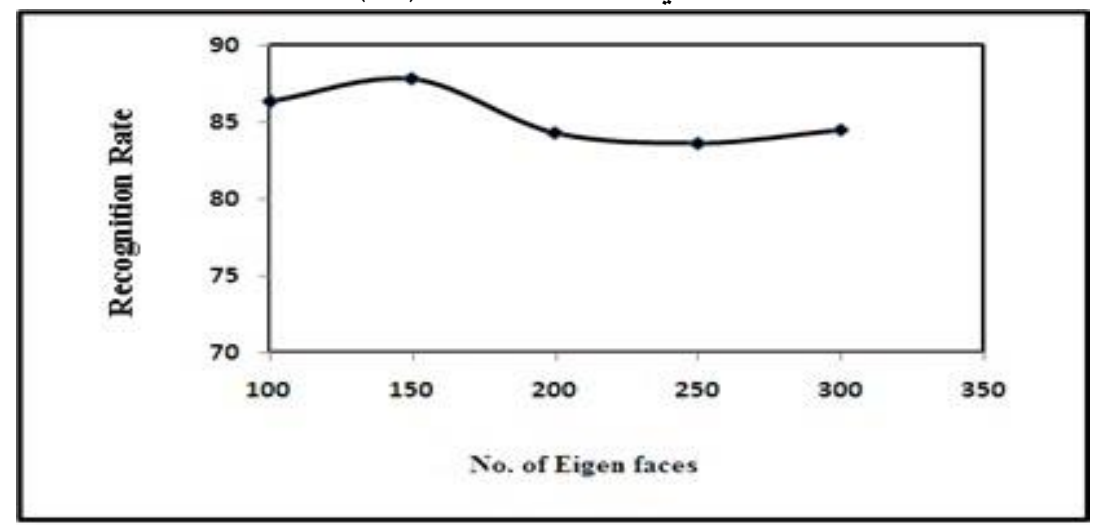

الثكل (13) نسب التمييز الآني لطريقة PCA+NN في التجربة الثانية

\section{PCA +LDA+NN \$ متائج تطبيق الـ}

تم حساب نسبة التمييز في حالة اختيار (300,250,200,150,100) على التوالي من متجهات الايكن في الـ PCA،ولكل حالة تم اختيار قيم معينة من متجهات الايكن في الـ LDA ونسبة التمييز في كل حالة تم حسابها و توضيحها بالجدول (2). 
جدول (2) نتائج التمييز الآني لطريقة PCA+LDA+NN في التجربة الثانية

\begin{tabular}{|c|c|c|c|c|c|}
\hline PCA & LDA & نسبة القبول & نسبة الرفض & وجه مكان آخر & نسبة التمييز \\
\hline \multirow{4}{*}{100} & 25 & 7.2993 & 4.3796 & 3.6496 & 84.6715 \\
\hline & 50 & 4.3478 & 1.4493 & 0.0 & 94.2029 \\
\hline & 75 & 4.3796 & 2.1897 & 0.0 & 93.4307 \\
\hline & 100 & 5.7554 & 3.5971 & 0.7194 & 89.9281 \\
\hline \multirow{3}{*}{150} & 50 & 6.2016 & 4.6512 & 1.5503 & 87.5969 \\
\hline & 100 & 5.4264 & 3.8759 & 0.0 & 90.6977 \\
\hline & 150 & 4.6512 & 2.3255 & 0.0 & 93.0233 \\
\hline \multirow{4}{*}{200} & 50 & 4.7244 & 3.9370 & 1.5748 & 89.7638 \\
\hline & 100 & 6.3492 & 4.7619 & 1.5873 & 87.3016 \\
\hline & 150 & 7.0313 & 4.6876 & 1.5623 & 86.7188 \\
\hline & 200 & 6.8702 & 4.5802 & 2.2901 & 86.2595 \\
\hline \multirow{5}{*}{250} & 50 & 5.3030 & 4.5455 & 1.5151 & 88.6364 \\
\hline & 100 & 5.4264 & 4.6512 & 1.5503 & 88.3721 \\
\hline & 150 & 5.1470 & 3.6765 & $\mathbf{0 . 7 3 5 3}$ & 90.4412 \\
\hline & 200 & 6.4516 & 4.8387 & 3.2258 & 85.4839 \\
\hline & 250 & 7.0313 & 5.4687 & 3.1250 & 84.3750 \\
\hline \multirow{6}{*}{300} & 50 & 5.6911 & 4.8781 & 1.6259 & 87.8049 \\
\hline & 100 & 7.2 & 6.4 & 3.2 & 83.2 \\
\hline & 150 & 5.3030 & 3.8031 & 1.5 & 89.3939 \\
\hline & 200 & 5.2239 & 3.7313 & 0.7463 & 90.2985 \\
\hline & 250 & 5.8823 & 4.4118 & 1.4706 & $\mathbf{8 8 . 2 3 5 3}$ \\
\hline & 300 & 5.9702 & 3.7314 & 1.4925 & 88.8059 \\
\hline
\end{tabular}

8. الاستنتاجات

بعد إتمام العمل واستعراض النتائج التي ظهرت من خلال التجارب والتطبيقات العملية على الخوارزميات المعتمدة في النظام المقترح تم التوصل إلى مجموعة من الاستتتاجات: • زيادة امكانية التمييز للوجه الواحد او اكثر ضمن اطار الفيديو في حالة الاعتماد على الاطار الرئيس.

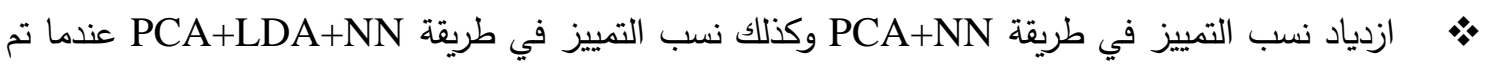

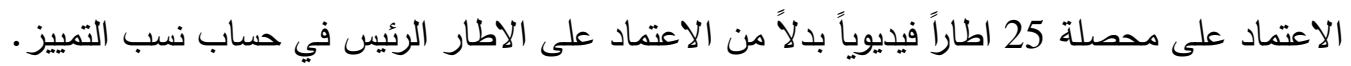

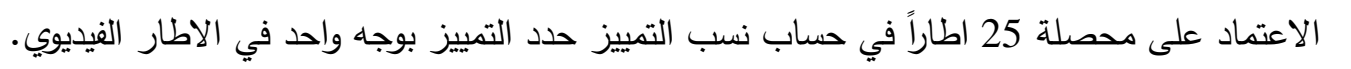
• أعلى نسب التمييز التي تم الحصول عليها في التجربتين سواءً عند تطبيق طريقة PCA وحدها في استخلاص الميزات أو عند تطبيق طريقة PCA+LDA هي عندما يتم اختيار عدد متجهات الايكن في خوارزمية PCA بين (40\% - 25\%) من عدد المتجهات الكلي الناتجة والتي تحمل أعلى قيمة أيكن. 


\section{المصادر}

[1] Gomathi E., Baskaran K., (2012), "Face Recognition Fusion Algorithm Based on Wavelet Transform and Fast Discrete Curvelet Transform", European Journal of Scientific Research, Vol. 74 , No. 3, pp: 450-455.

[2] Rúnarsson K., (2011), " A Face Recognition Plug-in for the PhotoCube Browser", M. Sc. PROJECT, School of Computer Science, Reykjavík University.

[3] Zhang J., Zhang X., Ha S., (2008), “A Novel Approach using PCA and SVM for Face Detection", proceedings of the 4th International Conference on Natural Computation, vol. 3, pp. 29-33.

[4] P. Latha, L. Ganesan, S. Annadurai, (2009), " Face Recognition using Neural Networks", signal processing : An International Journal (SPIJ), Vol. 3, Issue 5, PP:153-160.

[5] Agarwal M., Jain N., Kumar M., Agrawal H., (2010), "Face Recognition using Eigen Faces and Artificial Neural Network", International Journal of Computer Theory and Engineering (IJCTE), Vol. 2, No. 4, PP: 624-629.

[6] Wang-Xiaoping, (2011), "Face recognition based on bionic Pattern", International Conference on Electric Information and Control Engineering (ICEICE 2011), Vol. 1, PP: 2187-2189.

[7] Tofighi A., Monadjemi S. A., (2011), "Face Detection and Recognition Using Skin Color and AdaBoost Algorithm Combined with Gabor Features and SVM Classifier", International Conference on Multimedia and Signal Processing (CMSP), Vol. 1, PP:141-145.

[8] Yang M., Kriegman D. J., Ahuja N., (2002), " Detecting Faces in Images: A Survey", IEEE Transactions on Pattern Analysis and Machine Intelligence, Vol. 24, No. 1, PP:34-58.

[9] Verma M., Rani P., Kundra H., (2010), " A Hybrid Approach to Human Face Detection", International Journal of Computer Applications, Vol. 1, No. 13, PP:67-69.

[10] Kadoury S., (2005), " Face Detection Using Locally Linear Embedding”, M. Eng. thesis, Department of Electrical and Computer Engineering, McGill University, Montreal, Canada.

[11] Veerabhadrappa, Rangarajan L., (2010), "Multi-Level Dimensionality Reduction Methods Using Feature Selection and Feature Extraction", International Journal of Artificial Intelligence \& Applications (IJAIA), Vol. 1, No. 4, pp:54-68.

[12] Hasan H. M., AL-Jouhar W. A., Alwan M. A., (2012) "Face Recognition using improved FFT Based Radon by PSO and PCA Techniques", International Journal of Image Processing (IJIP), Vol. 6,Issue 1,PP: 26-37.

[13] Šochman J., (2004), “AdaBoost for Fast Face Detection”, Ph. D. Thesis, Faculty of Electrical Engineering, Czech Technical University.

[14] Viola P., Jones M. J., (2004), "Robust Real-Time Face Detection”, International Journal of Computer Vision, Vol. 57 , No. 2, PP:137-154. 
[15] Dong C., (2012), “Face Detection and Tracking using RGB-D Camera”, Master of Computing Thesis, College of Engineering \& Computer Science, Australian National University.

[16] Khan A., Farooq H., (2011), "Principal Component Analysis-Linear Discriminant Analysis Feature Extractor for Pattern Recognition", International Journal of Computer Science Issues(IJCSI), Vol. 8, Issue 6, No. 2, PP: 267-270.

[17] Subudhi K., Mishra R., (2011), " Human Face Detection and Recognition", Bachelor in Technology, Department of Electronics and Communication Engineering, National Institute of Technology, Rourkela.

[18] Hajraoui A., Sabri M., Bencharef O., Fakir M., (2012), "A New Approach for Face Recognition Based on PCA \& Double LDA Treatment combined with SVM", The Unit of International Organization of Scientific Research Journal of Engineering (IOSRJEN), Vol. 2, No. 4, pp:685-691.

[19] Eleyan A., Demirel H., (2007), "PCA and LDA based neural networks for Human Face Recognition", source: Face Recognition, Book edited by :Kresimir Delac and Mislav Grgic, PP:93-106, I-Tech Education and Publishing, Vienna, Austria.

[20] Wang X., (2002), "Feature Extraction and Dimensionality Reduction in Pattern Recognition and Their Application in Speech Recognition", Ph.D. thesis, Faculty of Engineering and Information Technology, Griffith University.

[21] More V., Wagh A., (2012), "Improved Fisher Face Approach for Human Recognition System using Facial Biometrics", International Journal of Information and Communication Technology Research, Vol. 2, No. 2, pp:135139.

[22] Bouzalmat A., Belghini N., Zarghili A., Kharroubi J., Majda A., (2011), "Face Recognition Using Neural Network Based Fourier Gabor Filters \& Random Projection", International Journal of Computer Science and Security (IJCSS), Vol. (5) , Issue (3), pp:376-386.

$$
\text { العصبية", رسالة ماجستير , قصم عليمة إحد, (2010), "التحقيق في كثف الحاسوب, كلية علوم التطفل بالاعتماد على الثبكة }
$$

[24] Li J., Cheng J., Shi J., Huang F., (2012), "Brief Introduction of Back Propagation (BP) Neural Network Algorithm and Its Improvement", Advances in Computer Science and Information Engineering (CSIE), Vol. 2, AISC 169, PP:553-558. 